hep-th/0404209

\title{
On The Classical Dynamics Of Charges In Noncommutative QED
}

\author{
Amir H. Fatollahi ${ }^{1}$ and Hossein Mohammadzadeh \\ Institute for Advanced Studies in Basic Sciences (IASBS), \\ P. O. Box 45195, Zanjan 159, Iran \\ fatho@mail.cern.ch \\ mohammad@iasbs.ac.ir
}

\begin{abstract}
One approach for formulating the classical dynamics of charged particles in nonAbelian gauge theories is due to Wong. Following Wong's approach, we derive the classical equations of motion of a charged particle in $U(1)$ gauge theory on noncommutative space, the so-called noncommutative QED. In the present use of the procedure, it is observed that the definition of mechanical momenta should be modified. The derived equations of motion manifest the previous statement about the dipole behavior of charges in noncommutative space.
\end{abstract}

\footnotetext{
${ }^{1}$ Address after March 2004: Department of Physics, Alzahra University, Tehran, 19938-91167, Iran
} 
In the last years much attention has been paid to the formulation and study of field theories on noncommutative spaces. Apart from the abstract mathematical interests, there are various physical motivations for doing so. One of the original motivations has been to get "finite" field theories via the intrinsic regularizations which are encoded in some of noncommutative spaces [1]. The other motivation comes from the unification aspects of theories on noncommutative spaces. These unification aspects have been the result of the "algebraization" of "space, geometry and their symmetries" via the approach of noncommutative geometry [2]. Interpreting the Higgs fields of the theories with spontaneously broken symmetries as gauge fields in the discrete directions of multi-sheet spaces is an example of this point of view on noncommutative spaces [3]. The other motivation refers to the natural appearance of noncommutative spaces in some areas of physics, and the recent one in string theory. It has been understood that string theory is involved by some kinds of noncommutativities; two examples are: (1) the coordinates of bound states of $N$ D-branes are represented by $N \times N$ Hermitian matrices [4], and (2) the longitudinal directions of D-branes in the presence of a B-field background appear to be noncommutative, as seen by the ends of open strings $[5,6,7]$. In the latter case, we encounter the spacetime in which the coordinates satisfy the canonical commutation relation

$$
\left[\hat{x}^{\mu}, \hat{x}^{\nu}\right]=i \theta^{\mu \nu},
$$

in which $\theta^{\mu \nu}$ is a constant second rank tensor. Since the coordinates do not commute, any definition of functions or fields should be performed under a prescription for ordering of coordinates, and a natural choice can be the symmetric one, the so-called Weyl ordering. To any function $f(x)$ on ordinary space, one can assign an operator $\hat{O}_{f}$ by

$$
\hat{O}_{f}(\hat{x}):=\frac{1}{(2 \pi)^{n}} \int d^{n} k \tilde{f}(k) \mathrm{e}^{-i k \cdot \hat{x}}
$$

in which $\tilde{f}(k)$ is the Fourier transform of $f(x)$ defined by

$$
\tilde{f}(k)=\int d^{n} x f(x) \mathrm{e}^{i k \cdot x} .
$$

Due to presence of the phase $\mathrm{e}^{-i k \cdot \hat{x}}$ in definition of $\hat{O}_{f}$, we recover the Weyl prescription for the coordinates. In a revers way we also can assign to any symmetrized operator a function or field living on the noncommutative plane. Also, we can assign to product of any two operators $\hat{O}_{f}$ and $\hat{O}_{g}$ another operator as

$$
\hat{O}_{f} \cdot \hat{O}_{g}=: \hat{O}_{f \star g}
$$


in which $f$ and $g$ are multiplied under the so-called $\star$-product defined by

$$
(f \star g)(x)=\left.\mathrm{e}^{\frac{i}{2} \theta^{\mu \nu} \frac{\partial}{\partial x^{\mu}} \frac{\partial}{\partial y^{\nu}}} f(x) g(y)\right|_{y=x}
$$

By these all one learns how to define physical theories in noncommutative spacetime, and eventually it appears that the noncommutative field theories are defined by actions that are essentially the same as in ordinary spacetime, with the exception that the products between fields are replaced by $\star$-products; see [8] as review. Though $\star$-product itself is not commutative (i.e., $f \star g \neq g \star f)$ the following identities make some of calculations easier in field theories:

$$
\begin{aligned}
& \int(f \star g)(x) d^{n} x=\int(g \star f) d^{n} x=\int(f \cdot g)(x) d^{n}(x) \\
& \int(f \star g \star h)(x) d^{n} x=\int(f \cdot(g \star h))(x) d^{n} x=\int((f \star g) \cdot h)(x) d^{n} x \\
& \int(f \star g \star h)(x) d^{n} x=\int(h \star f \star g)(x) d^{n} x=\int(g \star h \star f)(x) d^{n} x
\end{aligned}
$$

By the first two ones we see that, in integrands always one of the $\star$ 's can be removed.

The noncommutative QED (NCQED) is given by the action

$$
S=\int d^{4} x\left(-\frac{1}{4} F_{\mu \nu} F^{\mu \nu}-\bar{\psi} \gamma^{\mu}\left(\partial_{\mu}-i g A_{\mu^{\star}}\right) \psi-\frac{i m c}{\hbar} \bar{\psi} \psi\right)
$$

in which field strength is defined by

$$
F_{\mu \nu}=\partial_{\mu} A_{\nu}-\partial_{\nu} A_{\mu}-i g\left[A_{\mu}, A_{\nu}\right]_{M B}
$$

in which the commutator is that by Moyal, defined by

$$
\left[A_{\mu}, A_{\nu}\right]_{M B}=A_{\mu} \star A_{\nu}-A_{\nu} \star A_{\mu}
$$

The action is invariant under the transformations

$$
\begin{aligned}
& \psi \longrightarrow \psi^{\prime}=U \star \psi \\
& \bar{\psi} \longrightarrow \bar{\psi}^{\prime}=\bar{\psi} \star U^{-1} \\
& A_{\mu} \longrightarrow A^{\prime}{ }_{\mu}=U \star A_{\mu} \star U^{-1}+\frac{i}{g} U \star \partial_{\mu} U^{-1}
\end{aligned}
$$

in which $U(x)$ is the $\star$-phase $\left(U \star U^{-1}=U^{-1} \star U=1\right)$ defined by a function $\lambda(x)$ via the $\star$-exponential:

$$
U(x)=\exp _{\star}(i \lambda)=1+i \lambda-\frac{1}{2} \lambda \star \lambda+\cdots
$$


Under the gauge transformation, the field strength transforms as

$$
F_{\mu \nu} \longrightarrow F_{\mu \nu}^{\prime}=U \star F_{\mu \nu} \star U^{-1}
$$

We mention that the transformations of gauge fields as well as the field strength look like to those of non-Abelian gauge theories. Besides, we see that the pure gauge field sector of action contains terms which are responsible for interaction between the gauge particles, again as the situation we have in non-Abelian gauge theories.

Among others, there is one approach due to Wong [9] for derivation of the classical equations of motion of particles that have non-Abelian charges. In this formulation there are a couple of equations among which one is for the dynamics of the charged particle in spacetime, and one for the dynamics of isospin charge of particle, as an internal degrees of freedom. The former is analogous to Lorentz force in electro-magnetism. Noting the non-Abelian nature of NCQED, it is quite reasonable to use the approach by Wong to derive the classical equations of motion for charges in NCQED.

Let us review shortly Wong's approach in the next lines; see [10] ${ }^{2}$. The equations of motion for the fermionic matter field in fundamental representation and in the presence of background field $A_{\mu}(x)$ is:

$$
\gamma^{\mu}\left(\partial_{\mu}-i g A_{\mu}^{a} \hat{T}_{a}\right) \Psi(x)+\frac{i m c}{\hbar} \Psi(x)=0
$$

in which $\hat{T}_{a}$ 's are the generators of group, satisfying $\left[\hat{T}_{a}, \hat{T}_{b}\right]=i f_{a b}^{c} \hat{T}_{c}$. Viewing this equation as a Schrodinger equation (recalling $\partial_{0}=c^{-1} \partial_{t}$ ) one reads the Hamiltonian as

$$
\hat{H}=c \alpha^{i}\left(\hat{p}_{i}-g \hbar A_{i}^{a}(\hat{x}) \hat{T}_{a}\right)+m c^{2} \beta-g c \hbar A_{0}^{a}(\hat{x}) \hat{T}_{a}
$$

in which $\alpha^{i}$ and $\beta$ are the Dirac matrices, and $\hat{p}_{i}$ is sitting for $-i \hbar \partial_{i}$. In the Heisenberg picture, one obtains the equations of motion for operators as:

$$
\begin{aligned}
\dot{\hat{x}}^{i} & =\frac{i}{\hbar}\left[\hat{H}, \hat{x}^{i}\right]=c \alpha_{i}, \\
\dot{\hat{p}}_{i} & =\frac{i}{\hbar}\left[\hat{H}, \hat{p}_{i}\right]=g c \hbar\left(\alpha^{j} \partial_{i} A_{j}^{a}+\partial_{i} A_{0}^{a}\right) \hat{T}_{a}, \\
\dot{\hat{T}}_{a} & =\frac{i}{\hbar}\left[\hat{H}, \hat{T}_{a}\right]=-g f_{a b}^{c}\left(\dot{\hat{x}}_{i} A_{i}^{b}+c A_{0}^{b}\right) \hat{T}_{c}
\end{aligned}
$$

By defining the mechanical momenta by $\hat{\pi}_{i}:=\hat{p}_{i}-g \hbar A_{i}(\hat{x})$, one then gets

$$
\dot{\hat{\pi}}_{i}=g \hbar\left(c F_{i 0}^{a}+\dot{\hat{x}}^{j} F_{i j}^{a}\right) \hat{T}_{a}
$$

\footnotetext{
${ }^{2}$ Note missing $i=\sqrt{-1}$ in before of mass term in [10].
} 
in which the $F_{\mu \nu}$ 's are the field strength of the non-Abelian gauge theory, defined by $F_{\mu \nu}^{a}=\partial_{\mu} A_{\nu}^{a}-\partial_{\nu} A_{\mu}^{a}-g f_{b c}^{a} A_{\mu}^{b} A_{\nu}^{c}$. By comparison this equation with that of electromagnetism Wong suggests the following for the non-Abelian case:

$$
m \ddot{\xi}_{\mu}=g\left(F_{\mu \nu}^{a} T_{a}\right) \dot{\xi}^{\nu}
$$

in which $\xi^{\mu}(\tau)$ represents the world-line of the particle, and we used the change $\hbar \hat{T}_{a} \rightarrow T_{a}$. The dot in the equation is for derivative with respect to the proper-time. We mention that in the above equation $T_{a}$ 's can not be and are not supposed to be operators (i.e., matrices) anymore, while we interpret them as number functions capturing the degrees of freedom coming from group structure, satisfying the equations of motion:

$$
\dot{T}_{a}+g \dot{\xi}^{\mu} f_{a b}^{c} A_{\mu}^{b} T_{c}=0 .
$$

From this we learn that the group degrees of freedom, also known as isotopic spin, performs a precessional motion: $d / d \tau\left(T_{a} T^{a}\right)=0$.

Now we use Wong's method for the case of NCQED, and we do this in the first order of noncommutativity parameter $\theta_{\mu \nu}$. The Lagrangian in this order is:

$$
L=-\frac{1}{4} F^{\mu \nu} F_{\mu \nu}-\bar{\psi} \gamma^{\mu}\left(\partial_{\mu}-i g A_{\mu}\right) \psi-\frac{1}{2} g \bar{\psi} \gamma^{\mu} \theta^{\alpha \beta} \partial_{\alpha} A_{\mu} \partial_{\beta} \psi-\frac{i m c}{\hbar} \bar{\psi} \psi+O\left(\theta^{2}\right)
$$

in which the field strength is

$$
F_{\mu \nu}=\partial_{\mu} A_{\nu}-\partial_{\nu} A_{\mu}+g \theta^{\alpha \beta} \partial_{\alpha} A_{\mu} \partial_{\beta} A_{\nu}+O\left(\theta^{2}\right) .
$$

The action corresponding to the Lagrangian (23) is invariant under the first-order transformations in $\theta^{3}$ :

$$
\begin{aligned}
\psi & \longrightarrow \psi^{\prime}=\mathrm{e}^{i \lambda}\left(\psi-\frac{\theta^{\mu \nu}}{2} \partial_{\mu} \lambda \partial_{\nu} \psi\right)+O\left(\theta^{2}\right) \\
\bar{\psi} & \longrightarrow \bar{\psi}^{\prime}=\mathrm{e}^{-i \lambda}\left(\bar{\psi}-\frac{\theta^{\mu \nu}}{2} \partial_{\mu} \lambda \partial_{\nu} \bar{\psi}\right)+O\left(\theta^{2}\right) \\
A_{\mu} & \longrightarrow A^{\prime}{ }_{\mu}=A_{\mu}+\frac{1}{g} \partial_{\mu} \lambda-\theta^{\alpha \beta} \partial_{\alpha} \lambda \partial_{\beta} A_{\mu}-\frac{\theta^{\alpha \beta}}{2 g} \partial_{\alpha} \lambda \partial_{\beta} \partial_{\mu} \lambda+O\left(\theta^{2}\right)
\end{aligned}
$$

The equation of motion for $\psi$ is obtained to be:

$$
\gamma^{0}\left(\partial_{0}-i g A_{0}\right) \psi+\gamma^{i}\left(\partial_{i}-i g A_{i}\right) \psi+\frac{1}{2} g \gamma^{\mu} \theta^{\alpha \beta} \partial_{\alpha} A_{\mu} \partial_{\beta} \psi+\frac{i m c}{\hbar} \psi=0
$$

\footnotetext{
${ }^{3}$ The first-order transformations can be obtained by noting the fact that the $\star$-power of a function $f(x)$ behaves: $f_{\star}^{n}:=f \star f \star \cdots \star f=f^{n}+O\left(\theta^{2}\right)$, and hence we have $U(x)=\exp _{\star}(i \lambda)=\mathrm{e}^{i \lambda}+O\left(\theta^{2}\right)$.
} 
Here after we assume that noncommutativity is just for spatial directions: $\theta^{0 \mu}=\theta^{\mu 0}=0$. So, the above equation appears in the form:

$$
\gamma^{0}\left(\partial_{0}-i g A_{0}\right) \psi+\gamma^{i}\left(\partial_{i}-i g A_{i}\right) \psi+\frac{1}{2} g \theta^{i j} \gamma^{\mu} \partial_{i} A_{\mu} \partial_{j} \psi+\frac{i m c}{\hbar} \psi=0
$$

Again viewing this as a Schrodinger equation we read the corresponding Hamiltonian as

$$
\hat{H}=-g c \hbar A_{0}+c \alpha^{i}\left(\hat{p}_{i}-g \hbar A_{i}\right)+\frac{1}{2} g c \theta^{i j} \alpha^{\mu} \partial_{i} A_{\mu} \hat{p}_{j}+m c^{2} \beta
$$

in which we have used $\alpha^{\mu}=\left(\alpha^{0}, \alpha^{k}\right)=\left(I, \alpha^{k}\right)$. The Heisenberg equations of motion are derived for the operators as well:

$$
\begin{aligned}
& \dot{\hat{x}}^{l}=\frac{i}{\hbar}\left[\hat{H}, \hat{x}^{l}\right]=c \alpha^{l}+\frac{1}{2} g c \theta^{i l} \alpha^{\mu} \partial_{i} A_{\mu} \\
& \dot{\hat{p}}_{l}=\frac{i}{\hbar}\left[\hat{H}, \hat{p}_{l}\right]=g c \hbar \alpha^{\mu} \partial_{l} A_{\mu}-\frac{1}{2} g c \theta^{i j} \alpha^{\mu} \partial_{l} \partial_{i} A_{\mu} \hat{p}_{j}
\end{aligned}
$$

From the first equation we have $c \alpha^{l}=\dot{\hat{x}}^{l}-\frac{1}{2} g c \theta^{i l} \partial_{i} A_{0}-\frac{1}{2} g \theta^{i l} \dot{\hat{x}}^{k} \partial_{i} A_{k}+O\left(\theta^{2}\right)$; this will be used for later replacements. For the case of NCQED, we see that the interaction between fermions and gauge fields is different in comparison with ordinary (Abelian and non-Abelian) gauge theories. For the present case we have the following as mechanical momenta

$$
\hat{\pi}_{l}=\hat{p}_{l}-g \hbar A_{l}(\hat{x})+\frac{1}{2} g \theta^{i j} \partial_{i} A_{l}(\hat{x}) \hat{p}_{j}
$$

This form of mechanical momenta can be read also from the covariant derivative of NCQED, $D_{\mu} \psi=\partial_{\mu} \psi-i g A_{\mu} \star \psi$, which changes by similarity transformation under gauge transformations. After this modification to the Wong's approach, one can calculate the time derivative as

$$
\dot{\hat{\pi}}_{l}=\dot{\hat{p}}_{l}-g \hbar \partial_{t} A_{l}-i g\left[\hat{H}, A_{l}\right]+\frac{1}{2} g \theta^{i j} \partial_{t} \partial_{i} A_{l} \hat{p}_{j}+\frac{1}{2} g \theta^{i j} \frac{i}{\hbar}\left[\hat{H}, \partial_{i} A_{l} \hat{p}_{j}\right]
$$

After sufficient manipulations and replacements, and omitting hats we obtain

$$
\begin{aligned}
\dot{\pi}_{l} & =g c \hbar\left(\partial_{l} A_{0}-\partial_{0} A_{l}\right)+g \hbar \dot{x}^{i}\left(\partial_{l} A_{i}-\partial_{i} A_{l}\right) \\
& +\frac{1}{2} g c \theta^{i j} p_{j}\left(\partial_{0} \partial_{i} A_{l}-\partial_{l} \partial_{i} A_{0}\right)+\frac{1}{2} g \theta^{i j} \dot{x}^{k} p_{j}\left(\partial_{k} \partial_{i} A_{l}-\partial_{l} \partial_{i} A_{k}\right) \\
& -\frac{1}{2} g^{2} c \hbar \theta^{i j}\left(\partial_{i} A_{0}+\frac{1}{c} \dot{x}^{k} \partial_{i} A_{k}\right)\left(\partial_{j} A_{l}+\partial_{l} A_{j}\right)+O\left(\theta^{2}\right)
\end{aligned}
$$

By defining the field strengths:

$$
\begin{aligned}
& f_{\mu \nu}=\partial_{\mu} A_{\nu}-\partial_{\nu} A_{\mu} \\
& F_{\mu \nu}=f_{\mu \nu}+g \theta^{i j} \partial_{i} A_{\mu} \partial_{j} A_{\nu}
\end{aligned}
$$


and by setting $g \hbar c=e$ and $\mu^{i}:=\frac{1}{2} g c \theta^{i j} p_{j}=\frac{e}{2 \hbar} \theta^{i j} p_{j}$, we get

$$
\begin{aligned}
\dot{\pi}_{l} & =e\left(F_{l 0}+\frac{1}{c} \dot{x}^{i} F_{l i}\right) \\
& +\mu^{i} \partial_{i} f_{0 l}+\frac{1}{c} \mu^{i} \dot{x}^{k} \partial_{i} f_{k l}+\frac{1}{c} \dot{\mu}^{i} f_{l i}+O\left(\theta^{2}\right)
\end{aligned}
$$

The first two terms are easily understood as the dynamics of a charged particle in the background of noncommutative field strength $F_{\mu \nu}$. To understand the other terms, we compare the result with those of a dipole electric in the background of ordinary electromagnetic fields. The corresponding Lagrangian for a point-like electric dipole can be easily derived by considering the dynamics of two equal mass particles with opposite charges $q$ and $-q$, while their relative distance $\ell$ is small, defining the electric dipole $\vec{\mu}:=q \vec{\ell}$. So the starting point is

$$
L=\frac{1}{2} m \dot{\vec{x}}^{2}+\frac{1}{c} \mu^{i} \dot{x}^{i} f_{j i}+\mu^{i} f_{i 0}
$$

So the equation of motion for the position of dipole appears as

$$
m \ddot{x}_{k}=\mu^{j} \partial_{k} f_{0 j}-\frac{1}{c} \dot{\mu}^{j} f_{j k}+\frac{1}{c} \mu^{j} \dot{x}^{i}\left(\partial_{k} f_{j i}-\partial_{i} f_{j k}\right) .
$$

After using the Maxwell equation of ordinary electro-magnetism ${ }^{4}$, one ends up with the equation like that for charges in NCQED.

The result here by the Wong's approach in arbitrary background field has been pointed out also via the behavior of open strings ending on D-branes in presence of B-field [11], and also obtained through the studying of implications of possible noncommutativity in the present world in some specific examples $[12,13]$. From the string theory point of view the situation can be described as below. For example, the mode expansion of open string coordinates ending on a D2-brane is given by [11]:

$$
\begin{aligned}
& X^{0}=x^{0}+p^{0} \tau+\sum_{n \neq 0} a_{n}^{0} \frac{e^{-i n \tau}}{n} \cos n \sigma \\
& X^{i}=x^{i}+\left(p^{i} \tau-B_{j}^{i} p^{j} \sigma\right)+\sum_{n \neq 0} \frac{e^{-i n \tau}}{n}\left(i a_{n}^{i} \cos n \sigma+B_{j}^{i} a^{j} \sin n \sigma\right), \quad i=1,2 \\
& X^{b}=x^{b}+p^{b} \tau+\sum_{n \neq 0} a_{n}^{b} \frac{e^{-i n \tau}}{n} \cos n \sigma, \quad b=3, \cdots, 9
\end{aligned}
$$

in which $B_{j}^{i}$ are components of the B-field background. Now we see that for even the case in which the oscillations are suppressed, the distance between the ends of open strings on

\footnotetext{
${ }^{4}$ And using the identity $\epsilon_{j i l} \partial_{k}-\epsilon_{j k l} \partial_{i}=\epsilon_{k i l} \partial_{j}-\epsilon_{k i j} \partial_{l}$, for $i, j, k, l=1,2,3$.
} 
the D2-brane is not zero, appearing to be $\Delta^{i}=X^{i}(\sigma=0, \tau)-X^{i}(\sigma=\pi, \tau)=\pi B_{j}^{i} p^{j}$, by which we expect a dipole behavior due to \pm charges we assign to the ends of oriented open strings $[4,11]$. This behavior of open strings has been suggestive to formulate a theory for fields of dipoles rather than for fields that their quanta are particles [14]. The rule of multiplication of fields for dipoles is reminiscent of the $\star$-product.

Acknowledgement: The author is grateful to M. M. Sheikh-Jabbari for a final reading of the manuscript.

\section{References}

[1] H. S. Snyder, "Quantized Space-Time," Phys. Rev. 71 (1947) 38; "The Electromagnetic Field In Quantized Space-Time," Phys. Rev. 72 (1947) 68.

[2] A. Connes, "Noncommutative Geometry," Academic Press (1994).

[3] A. Connes and J. Lott, "Particle Models And Noncommutative Geometry," Nucl. Phys. (Proc. Supp.) B18 (1990) 29; A. H. Chamseddine and J. Frohlich, "SO(10) Unification In Non-Commutative Geometry," Phys. Rev. D50 (1994) 2893, hepth/9304023; A. H. Chamseddine, G. Felder and J. Frohlich, "Grand Unification In Non-Commutative Geometry," Nucl. Phys. B395 (1993) 672, hep-ph/9209224.

[4] E. Witten, "Bound States Of Strings And p-Branes," Nucl. Phys. B460 (1996) 335, hep-th/9510135.

[5] N. Seiberg and E. Witten, "String Theory And Noncommutative Geometry," JHEP 9909 (1999) 032, hep-th/9908142.

[6] A. Connes, M. R. Douglas and A. Schwarz, "Noncommutative Geometry And Matrix Theory: Compactification On Tori," JHEP 9802 (1998) 003, hep-th/9711162; M. R. Douglas and C. Hull, "D-Branes And The Noncommutative Torus," JHEP 9802 (1998) 008, hep-th/9711165.

[7] H. Arfaei and M. M. Sheikh-Jabbari, "Mixed Boundary Conditions And Brane-String Bound States," Nucl. Phys. B526 (1998) 278, hep-th/9709054.

[8] M. R. Douglas and N. A. Nekrasov, "Noncommutative Field Theory," Rev. Mod. Phys. 73 (2001) 977, hep-th/0106048. 
[9] S. K. Wong, "Field And Particle Equations For The Classical Yang-Mills Field And Particles With Isotopic Spin," Nuovo Cim. A65 (1970) 689.

[10] M. Carmeli, K. Huleihil and E. Leibowitz, "Gauge Fields: Classification And Equations Of Motion," World Scientific (1989), chap. 7.

[11] M. M. Sheikh-Jabbari, "Open Strings In A B-Field Background As Electric Dipoles," Phys. Lett. B455 (1999) 129, hep-th/9901080.

[12] M. Chaichian, M. M. Sheikh-Jabbari and A. Tureanu, "Hydrogen Atom Spectrum And The Lamb Shift In Noncommutative QED," Phys. Rev. Lett. 86 (2001) 2716, hep-th/0010175.

[13] I. F. Riad and M. M. Sheikh-Jabbari, "Noncommutative QED And Anomalous Dipole Moments," JHEP 0008 (2000) 045, hep-th/0008132.

[14] K. Dasgupta and M. M. Sheikh-Jabbari, "Noncommutative Dipole Field Theories," JHEP 0202 (2002) 002, hep-th/0112064. 